\title{
Optimization of Response Characteristics for Direct Feed Drive System with Partial Least Square model
}

\author{
Lin Xiankun, Li Peixiang, Li tiantian \\ School of Mechanical Engineering, University of Shanghai for Science and Technology, Shanghai \\ 200093, PR China
}

Keywords: Servo parameters, Linear motor, PLS

\begin{abstract}
There are lots of advantages using linear motor in high speed direct feed drive system. Nevertheless the control system of linear motor is easily being disturbed because of the distinctive structure. To improve the response characteristics of linear motor feed drive system, this paper proposes a regression model of servo parameters and an optimization strategy for control systems. The proposed regression model is established based on Partial least squares to facilitate the control system optimization. The proposed optimization strategy enables the determination of servo parameters that lead to stability performance without many trial-and-error tuning processes. In addition, the proposed strategy avoids machine tool in a state of resonance and being damaged. The effectiveness of the proposed regression model and optimization strategy was verified in experiments using a self-construction direct feed axis test rig.
\end{abstract}

\section{Introduction}

Linear motors possess several features such as fast response, high positioning accuracy, no travel restriction, high efficient, and so on [1]. Especially in the domain of high-speed and high-precision motion, linear motor feed drive system, without intermediate drive link, is an ideal feed drive mechanism for direct drive technology [2]. However, the distinctive "zero transmission" mode of this direct feed drive system makes the flexible load, internal disturbances and external interference directly effect on the working platform without through intermediate drive link, which hinders a wide application of linear motor [3-4] and also leads great difficulty for the servo control [5].

Improving stability performance of direct feed axis is of contemporary interest: exploring the various components matching of feed axis e.g. in [6-7], analyzing the operating conditions of feed axis e.g. in [8-9], studying the feed axis effective control algorithms and control parameters e.g. in [10-11]. It can make the working axis avoid resonance frequency to optimize the component of feed axis and redesign the operating conditions. However, there is more trouble in above two ways. When the feed axis entered service its component and operating conditions having been determined. Comparatively, it can be much easier to change the operating characteristics of the feed axis by only adjusting the control parameters and there are the advantages of low cost and high efficient in this method.

In CNC system, adjusting control parameters aims for the broader 0dB bandwidth of Bode diagram mainly by tuning repeatedly speed loop gain constant and integral time constant [12]. But in the application of this method, there are many problems. Firstly, if there are multiple overshoot points, it is often unable to accurately determine the resonance point of the feed axis. Secondly, in the absence of fully understanding the mechanical properties of machine tool components, the debug interval of servo gain cannot confirm and can be easily to damage the device. Furthermore a great blindness in the adjusting of servo parameters will be exist, if there is no sufficient experience of servo parameters adjustment or lack of guidance rules.

In the servo parameter optimization research, servo parameter regression model [13-14] can give a suitable parameter setting intervals to avoid damage device and blindness. According to the servo system [15], there is a high correlation between the servo parameters of speed control loop and current control loop. Therefore, the Least squares (LS) multivariate linear regression analysis method is of poor application. Although learning regression models, such as BP algorithms [16], can establish a high accurate and robust regression model, there are still many deficiencies, such as 
a long modeling time, excessive reliance on learning samples and difficulty to analyze the variables effects on modeling. The Partial least squares (PLS) proposed by WOLD make a significant progress in resolving the issue of independent variable correlation. PLS sets multiple linear regression analysis, principal component analysis, canonical correlation analysis as a whole. When the arguments set internal has a high degree correlation, it can integrate and screen the arguments to eliminate the multiple correlation among arguments, so then to the greatest extent extract related information between independent and dependent variables. The method has been well used in the fields of economic statistics, chemical engineering, chemical analysis and life science.

This paper establishes a servo parameter regression model taking direct feed axis for target and to avoid damage device proposing a parameter optimization strategy which can provide a suggestion for improving stability performance of feed axis. This proposed method takes Partial Least Squares as inference calculation tool to screening pre-debugging servo parameters and determine the servo gain space, which provide a new method for the direct feed axis servo parameter optimization automation.

\section{PLS identification methodology}

\subsection{PLS identification}

To study the relationship between the dependent variable and the independent variable, assume there are $p$ independent variables, $x=\left\{x_{1}, x_{2}, \ldots x_{p}\right\}$ and $q$ dependent variables, $y=\left\{y_{1}, y_{2}, \ldots y_{q}\right\}$ and also $\mathrm{n}$ samples of data are observed, then two matrices can be formulated, $\mathbf{X}=\left[x_{1}, x_{2}, \ldots x_{p}\right]_{n \times p}$ and $\mathbf{Y}=\left[y_{1}, y_{2}, \ldots y_{q}\right]_{n \times q}$. Because of the different dimension of the variables, the algorithm is implemented after the data have been pre-processed, i.e. scaling around zero mean and unit variance and the mean-centered and properly scaled matrices are obtained, $\mathbf{E}$ and $\mathbf{F}$. The first principal component ${ }^{\mathbf{t}_{1}}$ extracted from $\mathbf{E}$, carrying the relevant information in matrix $\mathbf{F}$ (excluding correlations) represented by some function for predicting $\mathbf{F}$. This expression can be written as follows:

$$
\begin{aligned}
& \max \left(\operatorname{Cov}\left(\mathbf{t}_{1}, \mathbf{F}\right)\right)=\max \left(\sqrt{\operatorname{Var}\left(\mathbf{t}_{1}\right)} \mathrm{r}\left(\mathbf{t}_{1}, \mathbf{F}\right)\right) \\
& \mathbf{t}_{1}=\mathbf{E w}_{1} \quad\left\|\mathbf{w}_{1}\right\|=1
\end{aligned}
$$

The Lagrange multiplier method enables to derive $\mathbf{w}_{1}$ as follows. Calculate the maximum eigenvalues of the matrix $\mathbf{E}^{\mathrm{T}} \mathbf{F} \mathbf{F}^{\mathrm{T}} \mathbf{E}$. Vector $\mathbf{w}_{1}$ is the standard eigenvector. A PLS model with $\mathbf{t}_{1}$ is expressed as follows:

$$
\begin{aligned}
& \mathbf{E}=\mathbf{t}_{1} \mathbf{p}_{1}^{\mathrm{T}}+\mathbf{E}_{1} \\
& \mathbf{F}=\mathbf{t}_{1} \mathbf{r}_{1}^{\mathrm{T}}+\mathbf{F}_{1} \\
& \mathbf{p}_{1}=\frac{\mathbf{E}^{\mathrm{T}} \mathbf{t}_{1}}{\left\|\mathbf{t}_{1}\right\|^{2}} \quad \mathbf{r}_{1}=\frac{\mathbf{F}^{\mathrm{T}} \mathbf{t}_{1}}{\left\|\mathbf{t}_{1}\right\|^{2}}
\end{aligned}
$$

Regression coefficient vector is $\mathbf{p}_{\mathbf{1}}, \mathbf{r}_{\mathbf{1}}$ and $\mathbf{E}_{1}, \mathbf{F}_{1}$ is the residual.

Replace $\mathbf{E}, \mathbf{F}$ with $\mathbf{E}_{1}, \mathbf{F}_{1}$ and repeat above step to get the second principal component. This is performed until the number of required factors is extracted, $\mathbf{t}_{1}, \mathbf{t}_{2}, \cdots \mathbf{t}_{m}$ or the residual is below some threshold. Matrix $\mathbf{E}, \mathbf{F}$ is decomposed into models similar to principal components:

$$
\begin{aligned}
& \mathbf{E}=\mathbf{t}_{1} \mathbf{p}_{1}^{\mathrm{T}}+\mathbf{t}_{2} \mathbf{p}_{2}^{\mathrm{T}}+\cdots \mathbf{E}_{m} \\
& \mathbf{F}=\mathbf{t}_{\mathbf{1}} \mathbf{r}_{\mathbf{1}}^{\mathbf{T}}+\mathbf{t}_{\mathbf{2}} \mathbf{r}_{\mathbf{2}}^{\mathbf{T}}+\cdots \mathbf{F}_{\mathbf{m}}
\end{aligned}
$$


Since $\mathbf{t}_{1}, \mathbf{t}_{2}, \cdots \mathbf{t}_{\mathrm{m}}$ all is a linear combination of variable $\mathbf{E}(1), \mathbf{E}(2), \cdots \mathbf{E}(k)(k \leq p)$ in $\mathbf{E}$ set, hence the variables in the $\mathbf{F}$ set can also be expressed by $\mathbf{E}(1), \mathbf{E}(2) \cdots \mathbf{E}(k)$, where $\mathbf{F}$ is a variable set normalized about dependent variable. Then, get normalized regression equation:

$$
\mathbf{F}=\mathbf{E}_{1} \mathbf{B}_{1}+\mathbf{E}_{2} \mathbf{B}_{2}+\cdots \mathbf{E}_{k} \mathbf{B}_{k}+\mathbf{F}_{m}
$$

Into actual parameters,

$$
\mathbf{y}=\mathbf{b}_{0}+\mathbf{x}_{1} \mathbf{b}_{1}+\mathbf{x}_{2} \mathbf{b}_{2}+\cdots \mathbf{x}_{k} \mathbf{b}_{k}+\mathbf{y}_{m}
$$

\subsection{Component validation}

According to the theory of multiple regression analysis, the number of components is that which gives the lowest residual on the test data. If too many factors are chosen, then the prediction property of the model is poor, so a balance has to be made on when the error reduction is minimal compared with the increase in the number of components. Therefore, in order to improve the accuracy of PLS regression model, the components need further screened to distinguish data information and noise. By adding a new component in this paper, observe whether the prediction property of the model has been significantly improved to judge the effectiveness of the ingredient. The specific method is as follows:

Firstly, the sample $i$ removed, use the rest samples and use $h$ components to fit a regression equation, then, the sample $i$ drag-in the equation, and calculate the fitting value $\hat{f_{h(-i)}}$ and predict the error square sum PRESS $_{h}$ being expressed as:

$$
\mathrm{PRESS}_{h}=\sum_{i=1}^{n}\left(f_{i}-\hat{f_{h(-i)}}\right)^{2}
$$

Secondly, using the entire sample to fit the regression equation containing $h$ components, where $\hat{f}_{h i}$ represents the sample $i$ and $S S_{h}$ represents the error squares sum being expressed as:

$$
S S_{h}=\sum_{i=1}^{n}\left(f_{i}-\hat{f}_{h i}\right)^{2}
$$

In general, $P R E S S_{h}$ is greater than $S S_{h}$ and $S S_{h}$ is less than ${ }^{S S_{h-1}}$, where ${ }^{S S_{h-1}}$ represents fitting errors of using $(h-1)$ components equation including all sample; PRESS $_{h}$ uses $h$ component but it contains sample disturbance error. If $P R E S S_{h}$ is less than $S S_{h}$ in a certain extent, it is believed that adding a component $\mathbf{t}_{h}$ can improve the accuracy of the fitting equation. Validity can be expressed as a formula:

$$
Q_{h}^{2}=1-\frac{P E R S S_{h}}{S S_{h}}
$$

Finally, in the state $Q_{h}^{2} \geq 0.0975$, it is considered that the contribution of components $\mathbf{t}_{h}$ to regression identification is prominent, and the regression process needs to introduce the component. Conversely, abandon $\mathbf{t}_{h}$ and the process of extracting components is finished.

\subsection{Independent variable validation}

The factors that influence the dependent variable are very many, and some independent variables are the dominant factor, but some independent variables are not obvious. An effective regression model is required to include all independent variables relating with dependent variables, and reject the irrelevant arguments and keep regression coefficients stable. In this paper, the independent variable is selected by calculating the difference between the residual matrix and the dependent variable uncertainty. The specific method is as follows: 
Firstly calculate the uncertainty of the dependent variable according to the project requirements on the dependent variable parameters. In the same state, measure dependent variables $\mathrm{n}$ times. Assuming that the effect each independent variable on the dependent variable is similar and the dependent variable uncertainty can be expressed as

$$
u(x)=\frac{s\left(x_{k}\right)}{\sqrt{n}}
$$

Constant $s\left(x_{k}\right)$ is the standard experiment deviation. Secondly, remove the independent variable $x_{i}$ and calculate the regression equation, then propose the same number of components to get matrix $\mathbf{y}_{m(-i)}$ the new residual. Finally, in the state $\mathbf{y}_{m(-i)} \geq u(x)$, it is considered that the independent variable $x_{i}$ plays a leading role in dependent variables and the regression process needs to retain the independent variable. Conversely, abandon the independent variable $x_{i}$ and reestablish the PLS model.

\section{Servo parameters regression model \& optimization strategy}

\subsection{Servo parameters regression model}

The servo system which is driven by linear motor includes position control loop, speed control loop, current control loop, vector conversion link, current control voltage mode inverter and corresponding feedback element. Analysis shows that the servo parameters of the current control loop and the speed control loop determine the stability of the displacement output signal. Hence, the independent variables are selected as speed loop gain $K_{v}$, velocity loop integral time ${ }^{T_{v}}$, current loop gain $K_{i}$ and current loop integral time ${ }^{T_{i}}$. Currently the main way to observe the work of servo system is reading the Bode diagram 0 dB band width. Therefore, dependent variable chooses the first overshoot point frequency $f$ and amplitude response $A$.

Each dependent variable in the security interval sets 20 calibration points, and measured 20 groups corresponding dependent variable values by reading CNC system Bode diagram. Based on MATLAB, write a PLS analysis program, translate 20 groups data into the standardization data by the central standardization processing and at last do regression analysis. The validity of each component is analyzed by Eq. (10), and the uncertainty of the arguments and the residual matrix are calculated. Using the regression equation calculated the predicted value of the dependent variable, and compared with the sample data to determine the accuracy of the model. The servo parameters debugging standard is that the first overshoot point frequency is max and amplitude response is less than $3 \mathrm{~dB}$. Therefore, when the dependent variable frequency is max, see the dependent variable amplitude response equaling to $3 \mathrm{~dB}$ as limit value to predict each independent variable servo parameters value.

\subsection{Servo parameter optimization strategy}

In order to avoid the debugging blindness and damage device when optimizing the direct feed axis servo parameters, the following servo parameters optimization strategy is proposed:

Step 1, Collect samples to establish regression model, according to the servo parameters regression model proposed by this paper.

Step 2, Get each independent variable adjustable ranges and limits from the servo parameters regression model and set the servo parameters value in servo system.

Step 3, According to the dependent variable frequency $f$, set the corresponding electronic filter to adjust output current.

Repeat above step to increase the servo gain and adjust the servo parameters according to frequency band required to optimize. This is performed until promise the overshoot frequency band 
amplitude is no more than $3 \mathrm{~dB}$ or make feed axis in the working frequency band of the $0 \mathrm{~dB}$ bandwidth obtain the maximal broadband.

\section{Experimental tests \& results analysis}

\subsection{The experimental scheme}

In order to verify the servo parameters optimization strategy, an established experimental system is shown in figure 1 in this paper. Experiment system is composed of Linear motor test bench; Numerical Control system and data capture system. The motor is SIEMENS 1 FN3 linear motor. The Numerical Control system is SINUMERIK 840D system.

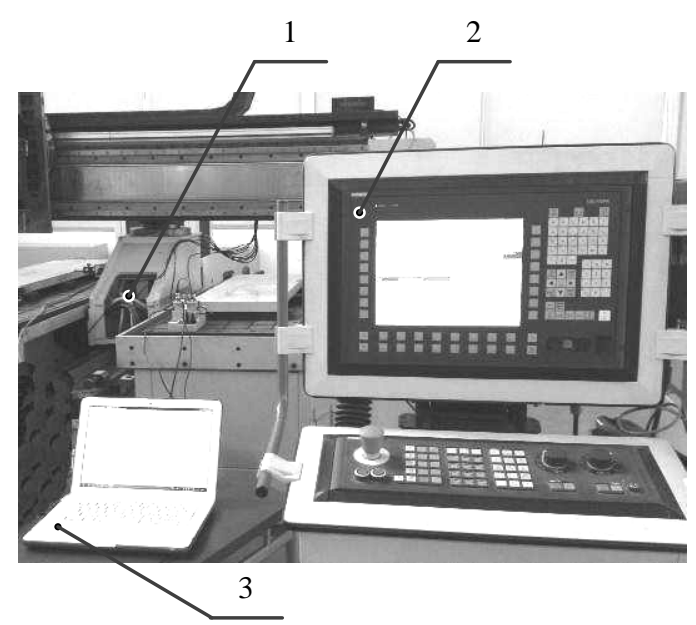

1. Linear motor test bench; 2. Numerical Control system; 3. Data capture system

Fig. 1 Experimental system

The corresponding variable servo parameters and variable adjustable range are defined in table 1. The independent variables are speed loop gain $K_{v}$, velocity loop integral time ${ }^{T_{v}}$, current loop gain $K_{i}$ and current loop integral time ${ }^{T_{i}}$.The dependent variables are the frequency of the first exceed adjusting point $f$ and amplitude response $A$.

Table 1 model parameter

\begin{tabular}{ccc}
\hline No & Variable & Interval \\
\hline 1 & $K_{v}(\mathrm{Ns} / \mathrm{m})$ & $10000 \sim 200000$ \\
2 & $T_{v}(\mathrm{~ms})$ & $0 \sim 30$ \\
3 & $K_{i}(\mathrm{~V} / \mathrm{A})$ & $30 \sim 50$ \\
4 & $T_{i}(\mu \mathrm{s})$ & $1000 \sim 6000$ \\
\hline 5 & $f(\mathrm{~Hz})$ & Observed value \\
6 & $A(\mathrm{~dB})$ & Observed value \\
\hline
\end{tabular}

According to the method mentioned above, establish the regression model of the servo parameters by 20 samples. The servo parameters extreme value is calculated by the regression model. Set the servo parameters of NC system and read the Bode diagram. Reset the servo gain value of NC system which is increased by $5 \%$ and read the Bode diagram again. Compare two diagrams and analyze the first overshoot point frequency and amplitude response.

\subsection{Results analysis}

Based on the calculation of servo parameters regression model, Fig. 2 shows the influence of servo parameters to the frequency response characteristic. Detailed analysis shows that: speed loop gain is the main servo parameters impacting the frequency response characteristics and the integration time plays a supplementary role. Furthermore, the coefficient of current loop gain is 
almost zero. In the case of the normal work of the current control loop, current loop gain has no obvious effect.

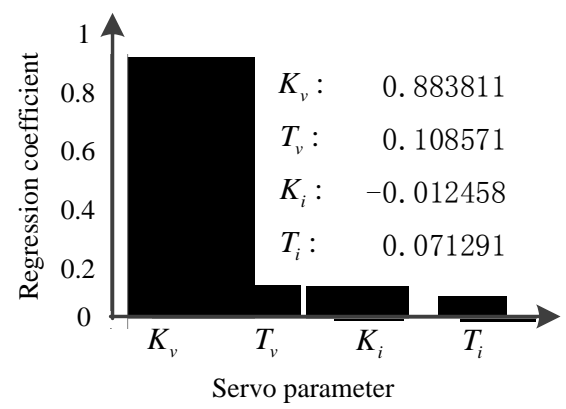

Fig. 2 The influence of servo parameters

According to the servo parameters of the regression model, the value of servo parameters can be predicted, $K_{v}$ is $192000 \mathrm{Ns} / \mathrm{m}, T_{v}$ is $0.1 \mathrm{~ms}, K_{i}$ is $48.7 \mathrm{~V} / \mathrm{A}$ and $T_{i}$ is $3500 \mu \mathrm{s}$. In figure 3, the Bode diagram I is measured by setting the corresponding servo parameters. Reset $K_{v}$ increasing 5\% to $201000 \mathrm{NS} / \mathrm{m}$ and measure the Bode diagram II. The Bode diagram I shows that in below $100 \mathrm{~Hz}$ there is no overshoot and The Bode diagram II shows that $7.5 \mathrm{~dB}$ overshoot is appeared at $19.5 \mathrm{~Hz}$.

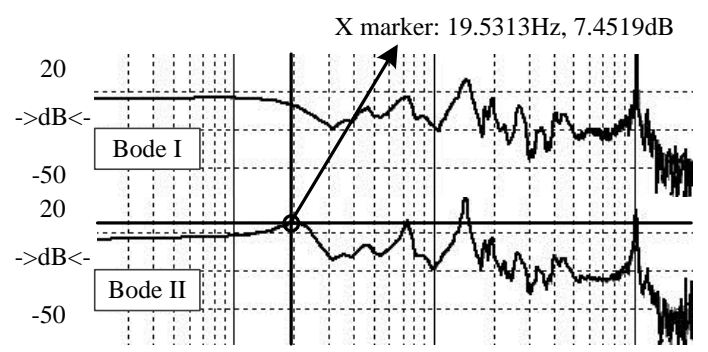

Fig. 3 Result comparison

In conclusion, the optimization strategy of servo parameters based on PLS regression in this paper is feasible. In condition of no limit test, the servo parameters optimal value is effectively determined and the stability of servo system is improved. In addition, when the servo parameters value increase 5\%, an overshoot point appears in Bode diagram.

\section{Conclusion}

A servo parameter regression model based on PLS is proposed and established. Analyze the influence of the independent variables on the dependent variables and make sure the main servo parameter in the process of debugging. According to the proposed servo parameters optimization strategy, a state of resonance didn't appear in the optimization process. Then make sure the safety of equipment and get the optimal value of the servo parameters.

\section{References}

[1] Wang Guobiao, Lai Yinan, Fan Dapeng et al. Summary of New Type Precision Transmission Design and Manufacture [J]. China Mechanical Engineering,2010,21(16): 1891 - 1897.

[2] Y. Altintas, A. Verl, C. Brecher et al. Machine tool feed drives [J]. Manufacturing Technology,2011,60: 779-796.

[3] Ma Ping, Chen Zhenhuan, Li Jieke et al. Dynamic stiffness of high velocity feed unit of the zero transmission machine tools [J]. China Mechanical Engineering,2004,15（7） : 575-577. 
[4] Chen Shangliang, Hsieh Tsunghsien. Repetitive control design and implementation for linear motor machine tool [J]. International Journal of Machine Tools \& Manufacture,2007(47): 18071816.

[5] Wang Mulan, Zhang Chongwei, Lin Jian et al. Development of an Experimental Platform for Linear Motors in CNC Machine Tools [J]. China Mechanical Engineering,2012,23(3): 274-278.

[6] M. Weck, D. Staimer. Parallel Kinematic Machine Tools-Current State and Future Potentials [J]. CIRP Annals, 2002(51):671-683.

[7] V.T. Portman, V.S. Chapsky, Y. Shneor. Evaluation and optimization of dynamic stiffness values of the PKMs: Collinear stiffness value approach [J]. Mechanism and Machine Theory,2014(74): 216-244.

[8] Mao Kuanmin, Li Bin, Wu Jun et al. Stiffness influential factors-based dynamic modeling and its parameter identification method of fixed joints in machine tools [J]. International Journal of Machine Tools \& Manufacture,2010(50): 156-164.

[9] MI Liang, Yin Guofu, Sun Mingnan et al. Effects of preloads on joints on dynamic stiffness of a whole machine tool structure [J]. Journal of Mechanical Science and Technology,2012,26(2): 495-508.

[10] Shen Binhong, Tsai Miching. Robust dynamic stiffness design of linear servomotor drives [J]. Control Engineering Practice, 2006,14: 1325-1336.

[11]Yen Jiayush, Chang Huiman. Performance robustness and stiffness analysis on a machine tool servo design [J]. International Journal of Machine Tools \& Manufacture,2004(44): 523-531.

[12] SIEMENS, SINUMERIK 840D Installation and Start-Up Guide [CD]. SIEMENS, 2006.

[13]Ren Yongjie, Zheng Jigui, Yang Xueyou, et al. Method of robot calibration based on laser tracker [J]. Chinese Journal of Mechanical Engineer, 2007, 43(9):195-200.

[14]Zhang Yonggui, Huang Yumei, Gao Feng, et al. New model for air spray gun of robotic spraypainting [J]. Chinese Journal of Mechanical Engineer, 2007, 43(9):195-200.

[15]Zhang Ying. Research on the Analysis of Detent Force and Control Strategies of Permanent Magnetic Synchronous Linear Motors [D].Wuhan : Huazhong University of Science \& Technology,2008: 70-84.

[16]Huang y q, Zhang j, Li x. Thermal error modeling by integrating GA and BP algorithms for the high-speed spindle [J]. International Journal Advanced Manufacturing Technology, 2014,71:16691675.

[17]CHANG Y J, LU X ZH, WANG SH L, et al. Study on the linearization of analyzer for engine exhaust based on partial least squares [J]. Journal of mechanical engineering, 2011, 47(10):76-81. 